

\title{
Etat structural, enracinement et alimentation hydrique du maïs. III. - Disponibilité des réserves en eau du sol
}

François Tardieu

\section{To cite this version:}

François Tardieu. Etat structural, enracinement et alimentation hydrique du maïs. III. - Disponibilité des réserves en eau du sol. Agronomie, 1987, 7 (4), pp.279-288. hal-00884992

\section{HAL Id: hal-00884992 \\ https://hal.science/hal-00884992}

Submitted on 1 Jan 1987

HAL is a multi-disciplinary open access archive for the deposit and dissemination of scientific research documents, whether they are published or not. The documents may come from teaching and research institutions in France or abroad, or from public or private research centers.
L'archive ouverte pluridisciplinaire HAL, est destinée au dépôt et à la diffusion de documents scientifiques de niveau recherche, publiés ou non, émanant des établissements d'enseignement et de recherche français ou étrangers, des laboratoires publics ou privés. 


\title{
Etat structural, enracinement et alimentation hydrique du maïs. III. - Disponibilité des réser- ves en eau du sol
}

François TARDIEU

I.N.R.A., Laboratoire d'Agronomie, Centre de Recherches de Paris-Grignon, F 78850 Thiverval-Grignon

Mots clés additionnels : Résistance stomatique, bilan hydrique du sol, variabilité spatiale, tassement du sol, transferts d'eau sol-plante, groupement des racines.

\begin{abstract}
Variations in soil water reserves and stomatal conductance of the upper leaves of maize canopies were monitored for 3 years during the 6-week period around silking. Measurements were carried out in plots of an experimental field where the ploughed layer structure was contrasted, and where root system and top growth were also studied. In compacted plots, water uptake was reduced nearly by half compared with other plots ; as a consequence, stomatal conductance was different between treatments after a few days without rain, although soil water content always remained higher in compacted plots compared with plots having a favourable structure. A correspondence between soil water content variations and the geometrical characteristics of root system showed : (1) that differences between treatments in the availabity of water reserves are probably due to the spatial distribution of roots, and (2) that part of the spatial variability of soil drying is linked with irregularities in this distribution.
\end{abstract}

Additional key words : Stomatal resistance, soil water balance, spatial variability, soil compaction, soilplant water transfers, root clumping.

\section{LISTE DES ABRÉVIATIONS}

H1 Horizon de la couche labourée, affiné lors du travail superficiel (de 0 à $10 \mathrm{~cm}$ ).

H5 Horizon non affiné de la couche labourée (de 10 à $28 \mathrm{~cm}$ ).

O Traitement expérimental : $\mathrm{H} 5$ de structure fragmentaire, formé de terre fine.

B Traitement expérimental : H5 de structure fragmentaire formé de mottes de plusieurs $\mathrm{dm}^{3}$ et de cavités structurales.
C Traitement expérimental : H5 de structure continue et compacte.

G82, G83, G85, M82 : Essais effectués à Grignon (G) ou à Montmirail (M) en 1982, 1983 ou 1985.

$\mathrm{n}$ « $\mathrm{f}$ » Stade phénologique « $\mathrm{n}$ feuilles visibles ».

$>$ Différence significative, comparaison de moyennes par le « $\mathrm{T}$ » de Bonferroni, risque global 5 p. 100.

$[\Delta \mathrm{S}]{ }^{\mathrm{z} 2}$ Variation de stock d'eau du sol dans la couche de z1 sol comprise entre les profondeurs $\mathrm{zl}$ et $\mathrm{z} 2(\mathrm{~mm})$. 


\section{INTRODUCTION}

L'existence d'un effet de l'état structural de la couche labourée sur l'alimentation hydrique du maîs est fortement suggéré par les observations de MANICHON \& SEBILloTTE (1973) et par des mesures d'humidité dans des sols travaillés (VAN LOON \& BOUMA, 1978 ; RAGHAVAN et al., 1979). D'après les conclusions de plusieurs travaux (NEWMAN, 1969 ; REICOSKY \& RITCHIE, 1976 ; ZUR et al., 1982), cet effet ne pourrait être lié qu'à la profondeur d'enracinement, qui délimite le volume de terre colonisé. Ces auteurs considèrent en effet que, à l'intérieur de ce volume, la résistance aux transferts d'eau vers les racines sont faibles, et que seules peuvent être appréciables la résistance au transfert entre couches non enracinées et enracinées, ou celle à l'intérieur des plantes.

Or, sur le dispositif de Grignon (Yvelines) où sur un même champ sont comparées des parcelles expérimentales d'états structuraux contrastées (TARDIEU \& MANICHON, 1987a), nous avons montré que la profondeur d'enracinement mesurée à la floraison est peu différente d'un traitement à l'autre (TARDIEU \& MANICHON, 1987b). La couche de terre atteinte par les racines et la " réserve utile » du sol sont donc semblables entre traitements, et seules diffèrent la densité racinaire et, surtout, la disposition spatiale des racines. Le but de cet article est d'analyser les conséquences sur les transferts d'eau sol-plante de ces différences d'enracinement, dues à l'état structural de la couche labourée.

Cette étude a été menée en 1982, 1983 et 1985, principalement pendant la période qui encadre la floraison du maîs, qui est la plus critique par rapport à l'élaboration du rendement (DENMEAD \& SHAW, 1960 ; ROBELIN, 1963 ; ClaASEN \& SHAW, 1970).

\section{MATÉRIEL ET MÉTHODES}

\section{A. Dispositif expérimental}

L'essai pluriannuel présenté ici a été mené avec le dispositif présenté dans le premier article de cette série (TARDIEU \& MANICHON, 1987a). Sur chacun des traitements $\mathrm{O}, \mathrm{B}$ et $\mathrm{C}$, le système racinaire a été caractérisé après la floraison par une méthode cartographique qui permet de calculer, d'une part, la fonction de répartition des distances entre chaque point du sol et la racine la plus proche et, d'autre part, un indice synthétique de caractérisation des systèmes racinaires en tant que capteurs d'eau. Celui-ci est fondé sur la disposition spatiale des racines et sur le type du contact entre celles-ci et la terre environnante (TARDIEU \& MANICHON, 1987b). Les indices foliaires ont été obtenus en 1983 et 1985 à 5 dates en prélevant 3 placettes de $1 \mathrm{~m}^{2}$ chacune par traitement, et en mesurant la surface des feuilles apparues avec un planimètre.

La période comprise entre les stades « $10 \mathrm{f}$ » et « floraison plus 3 semaines ", pendant laquelle l'étude la plus intensive a été effectuée, a été relativement pluvieuse en 1982 et 1983, au contraire de 1985 (tabl. 1; moyenne sur 20 ans des précipitations entre le 23.6 et le $5.8: 83 \mathrm{~mm}$ ). Lors des 3 années, les 2 semaines précédant la floraison ont été sèches, avec des ETP Piche élevées (formule de BOUCHET, appelée $\operatorname{ETP}_{p}$ dans la suite du texte) excédant souvent $5 \mathrm{~mm}$. jour ${ }^{-1}$.

\section{B. Caractérisation des états hydriques du sol et des peuplements végétaux}

Deux approches ont été adoptées pour cette caractérisation :

- la mesure répétée de la teneur en eau du sol sur chaque parcelle expérimentale, qui permet d'une part de comparer entre traitements la variation du stock d'eau du sol, et d'autre part d'étudier la variabilité horizontale et verticale de ce dessèchement, en liaison avec les caractéristiques des systèmes racinaires ;

- la mesure de la conductance stomatique (inverse de la résistance) des feuilles supérieures de chaque couvert végétal, destinée à évaluer sur des pas de temps courts le degré de satisfaction par le système racinaire des besoins en eau des peuplements de maïs. L'évaluation de ce degré de satisfaction à partir du seul bilan hydrique (BOUCHET \& ROBELIN, 1969) n'aurait pas en effet été suffisamment fiable pour notre étude, compte tenu de la grande variabilité spatiale de chacun des termes de ce bilan (VAUCLIN et al., 1984) et de l'imprécision sur certains de ceux-ci (DAUDET \& VACHAUD, 1977).

TABLEAU 1

Caractéristiques climatiques des périodes étudiées, position des périodes sans pluies et dates de réalisation des stades végétatifs. $10 f$ : stade " 10 feuilles visibles"; $F$ : émission des soies; $F+3 s: 3$ semaines après l'émission des soies.

Climatic characteristics of the studied periods, position of dry periods and dates of vegetative stages. $10 \mathrm{f}$ : stage '10 visible leaves'; $F:$ silking ; $F+3 s: 3$ weeks after silking.

\begin{tabular}{|c|c|c|c|c|c|c|c|c|}
\hline & \multirow{2}{*}{\multicolumn{4}{|c|}{ Stades végétatifs (trait. $O$ ) }} & & \multirow{2}{*}{\multicolumn{2}{|c|}{ Périodes sans pluie }} \\
\hline & & & & & $10 \mathrm{f}$ & $F+3 s$ & & \\
\hline & levée & $10 \mathrm{f}$ & $\mathbf{F}$ & $F+3 s$ & $\begin{array}{l}\text { Pluie } \\
(\mathrm{mm})\end{array}$ & $\begin{array}{l}\operatorname{ETP}_{p} \\
\text { cumulée }\end{array}$ & Dates & $\begin{array}{l}\mathrm{ETP}_{\mathrm{p}} \\
\text { cumulée }\end{array}$ \\
\hline 1982 & 23.5 & 16.6 & 12.7 & 3.8 & 142 & 142 & 3.7 au 14.7 & 37,3 \\
\hline 1983 & 23.5 & 23.6 & 14.7 & 5.8 & 110 & 140 & 7.7 au 18.7 & 52,5 \\
\hline 1985 & 17.5 & 24.6 & 16.7 & 7.8 & 62 & 182 & 24.6 au 13.7 & 66,7 \\
\hline
\end{tabular}


1. Suivi de la teneur en eau du sol et du potentiel hydrique des couches profondes

a) La teneur en eau du sol a été suivie en 1983 sur les traitements $\mathrm{O}, \mathrm{B}$ et $\mathrm{C}$, et en 1985 sur les traitements $\mathrm{O}$ et $C$, avec un pas de temps d'environ $4 \mathrm{j}$.

- Dans la couche labourée $(0-28 \mathrm{~cm})$, de 8 à 16 échantillons de $70 \mathrm{~cm}^{3}$ environ ont été prélevés dans les couches $0-3,3-9,9-15$ et $15-28 \mathrm{~cm}$, chaque échantillon étant également repéré par l'état structural de la zone d'où il provient (bloc tassé, peu ou mal enraciné ou zone meuble enracinée). L'humidité pondérale a été mesurée après dessication pendant $48 \mathrm{~h}$ à $105^{\circ} \mathrm{C}$, et transformée en humidité volumique en utilisant les masses volumiques mesurées (TARDIEU \& MANICHON, $1987 a$ ). En raison de sa lourdeur cette mesure n'a été effectuée qu'en début et en fin des périodes sans pluie.

- A partir de $15 \mathrm{~cm}$ de profondeur, l'humidité a été suivie, jusqu'à $175 \mathrm{~cm}$ avec un humidimètre neutronique NARDEUX Solo $20(40 \mathrm{mCi})$. Six tubes verticaux d'alliage AG 3 ont été mis en place par traitement (4 tubes en 1985), avant le stade 《 $5 \mathrm{f}$ » du maïs, et après une $1^{\text {re }}$ évaluation de l'état de croissance des parties aériennes. Les tubes ont été placés à $15 \mathrm{~cm}$ du rang de semis à l'intérieur de placettes où, dans les traitements $\mathrm{O}$ et $\mathrm{B}$, aucun tassement post-labour n'avait eu lieu (TARDIEU \& MANICHON, 1987a), et où la disposition des plantes et les états de croissance des pieds étaient aussi réguliers que possible. L'étalonnage de la sonde a été effectué par mesure de l'humidité pondérale dans des échantillons de terre prélevés au début de la campagne, lors de la pose des tubes, et à la fin de celle-ci à proximité immédiate des tubes. Une pente moyenne a été calculée pour chacun des horizons pédologiques, mais une ordonnée à l'origine particulière à chaque profondeur de chaque tube a été déterminée et prise en compte dans les calculs ultérieurs. Des mesures gravimétriques ont été faites en cours de campagne afin de vérifier l'exactitude de l'étalonnage.

b) Le calcul de la variation de stock d'eau a été effectué par tranches de sol $(0-3,3-9$ et $9-15 \mathrm{~cm}$, tous les $10 \mathrm{~cm}$ au-delà), et intégré sur 2 couches. La $1^{\text {re }}$ $\left([\Delta S]_{0}^{15}\right)$, de 0 à $15 \mathrm{~cm}$ de profondeur est constituée principalement de l'horizon $\mathrm{H} 1$, affiné dans tous les traitements lors du travail superficiel et dont l'enracinement est peu différent d'un traitement à l'autre (TARDIEU \& MANICHON, 1987b) ; elle comprend également les premiers $\mathrm{cm}$ de l'horizon labouré non repris (H5) situé à proximité des racines de l'horizon H1. La $2^{\mathrm{e}}$ couche considérée, $\left([\Delta S]^{175}\right)$ de 15 à $175 \mathrm{~cm}$, est constituée du reste de l'horizon $\mathrm{H} 5$ et des couches sous-jacentes, où de fortes différences d'enracinement ont été constatées.

c) En 1985, 3 batteries de tensiomètres ont été placées dans le traitement $O$ à respectivement 110, 130, 150,165 et $185 \mathrm{~cm}$ de profondeur.

d) Des courbes potentiel hydrique-humidité volumique du sol ont été construites pour chaque horizon pédologique (TARDIEU, 1984). Les mesures ont été effectuées sur des agrégats d'environ $30 \mathrm{~cm}^{3}$, avec un appareil de RICHARDS pour les potentiels inférieurs à -1 bar, et une table à succion pour les potentiels supérieurs.

\section{Conductance stomatique}

Celle-ci a été mesurée au cours de journées claires avec un poromètre à diffusion (LI-COR L.I. 700), en suivant le mode opératoire proposé par KATERJI \& OULID-AISSA (1983). Les mesures ont été faites approximativement toutes les heures, exprimées en temps solaire vrai (TSV) très proche à Grignon de l'heure légale - 2 pour la période considérée. L'objectif est ici de comparer l'alimentation hydrique des peuplements végétaux de chaque traitement, ce qui a eu 2 conséquences :

- nous avons choisi la conductance stomatique (inverse de la résistance) comme variable étudiée : sa variance est comparable d'un traitement à l'autre (condition nécessaire pour la réalisation de tests statistiques classiques), ce qui n'est pas le cas de la résistance pour laquelle on a observé des variances 1000 fois plus grandes en C par rapport à O (TARDIEU, 1984) ;

- nous n'avons pas cherché à mesurer la conductance dans chacune des strates des couverts étudiés (cf. KATERJI et al., 1983). Nous nous sommes limités à la strate supérieure, en y échantillonnant des feuilles de même âge physiologique (à partir du stade « $11 \mathrm{f}$ », la feuille choisie est celle située au-dessus de l'ébauche du premier épi), recevant des éclairements voisins (ce qui a nécessité de n'échantillonner, à chaque heure de la journée, que des feuilles ayant la même orientation par rapport au soleil). Les mesures ont été effectuées sur la face inférieure des feuilles où, chez le maïs, la conductance stomatique est plus sensible à une variation du potentiel hydrique (SANCHEZ-DIAZ \& KRAMER, 1971). Entre 20 et 40 feuilles de cette catégorie ont été échantillonnées à chaque heure dans 5 placettes de $1 \mathrm{~m}^{2}$ délimitées à l'intérieur de chaque parcelle expérimentale. Les cartographies racinaires effectuées à la floraison ont été faites sur ces mêmes placettes.

\section{RÉSULTATS}

\section{A. Utilisation des réserves en eau du sol}

Les valeurs de la teneur en eau du sol en début de cycle végétatif ont été publiées dans le premier article de cette série (TARDIEU \& MANICHON, 1987a). Nous ne présenterons et ne discuterons ici que les variations d'humidité au cours des périodes sans pluie.

1. Dans les 15 premiers cm de profondeur, la variation de stock d'eau entre le début et la fin des périodes sèches du mois de juillet est peu différente entre $O$ et $C$, avec une tendance vers un dessèchement plus faible en B (tabl. 2). Elle est plus grande en 1985 qu'en 1983, en relation avec ''ETP $_{\mathrm{p}}$ cumulée pendant les mêmes périodes. La variation de stock d'eau dans cette couche est de l'ordre du tiers à la moitié de celle de la couche $0-175 \mathrm{~cm}$.

2. Les variations de stock d'eau dans la couche 15 $175 \mathrm{~cm}$ sont présentées aux figures $1 a$ et $b$ pour les années 1983 et 1985 . Elles sont moins sensibles aux fluctuations climatiques que celles de l'horizon 0 - 
TABLEAU 2

Variation du stock d'eau du sol pendant les périodes sans pluie de 1983 et 1985. A l'intérieur d'une ligne et d'une année, les moyennes suivies d'une même lettre ne sont pas significativement différentes au seuil $5 \%$ dans une comparaison multiple de Bonferroni.

Variation in soil water reserves during dry periods in 1983 and 1985. Means within horizontal lines and year followed by the same letter are not significantly different at the $5 \%$ level according to Bonferroni's multiple test.

\begin{tabular}{|c|c|c|c|c|c|}
\hline & \multicolumn{3}{|c|}{1983} & \multicolumn{2}{|c|}{1985} \\
\hline & $\mathrm{O}$ & B & $\mathrm{C}$ & $\mathrm{O}$ & $\mathrm{C}$ \\
\hline$[\Delta \mathrm{S}]_{0}^{15}$ & $18,2 \mathrm{a}$ & $15,2 \mathrm{a}$ & $18,7 \mathrm{a}$ & $24,2 \mathrm{a}$ & $23.1 \mathrm{a}$ \\
\hline$[\Delta \mathrm{S}]_{[5}^{175}$ & $36,2 \mathrm{a}$ & $35,7 \mathrm{a}$ & $20,5 \mathrm{~b}$ & $43,7 \mathrm{a}$ & $19,7 \mathrm{~b}$ \\
\hline Total & $54,4 \mathrm{a}$ & $50,9 \mathrm{a}$ & $39,2 \mathrm{~b}$ & 67,9 a & $42,8 \mathrm{~b}$ \\
\hline \multicolumn{6}{|l|}{$[\Delta S]_{0}^{175}$} \\
\hline ETP & 1,04 & 0,97 & 0,75 & 1,02 & 0,64 \\
\hline
\end{tabular}

$15 \mathrm{~cm}$, bien que l'effet des précipitations apparaisse clairement (en particulier les 22.7 .83 et 2.8 .85 ). Une différence significative apparaît entre les traitements $\mathrm{O}$ et $\mathrm{C}$, à toutes les dates des périodes étudiées (tabl. 2) : à la fin des périodes sans pluie, la variation de stock d'eau est de l'ordre de la moitié en $\mathrm{C}$ par rapport à $\mathrm{O}$. En revanche, en 1983, les traitements O et B ne se différencient pas sur ce critère.

Les modalités du dessèchement du sol sont présentées à la figure 2 pour la période du 8 au 17.7.83. Dans la couche labourée de $B$ et $C$, les zones massives ont perdu significativement moins d'eau que les zones fragmentaires. Ceci se traduit par un dessèchement de l'horizon $\mathrm{H} 5$ très inférieur en $\mathrm{C}$ par rapport à $\mathrm{O}$; dans le traitement $B$ où ces 2 types de zones sont voisins dans le profil, les 2 comportements coexistent (fig. $2 b$ ). Les couches situées sous le fond du labour, bien que d'états structuraux identiques entre traitements (TARDIEU \& MANICHON, 1987a) sont dessèchées d'une
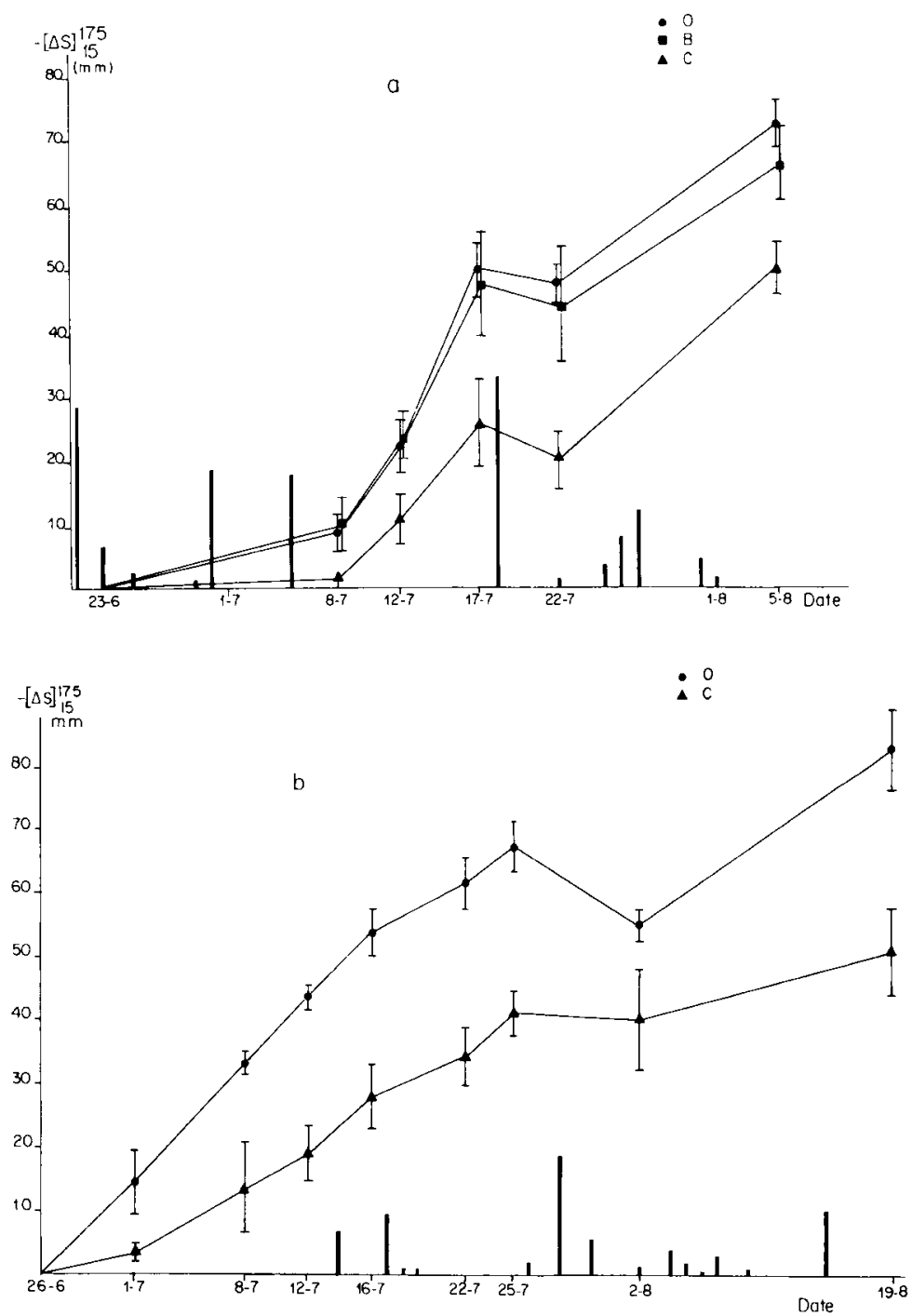

Figure 1

Evolution au cours du temps du stock d'eau du sol dans la couche 15 à $175 \mathrm{~cm}\left([\Delta \mathrm{S}]_{15}^{175}\right)$ et précipitations (traits verticaux) :

a) 1983, calculs effectués à partir du 23.6,

b) 1985, calculs effectués à partir du 26.6.

Variation of water reserves in the $15-175 \mathrm{~cm}$ layer of $\operatorname{soil}\left([\Delta \mathrm{S}]_{15}^{175}\right)$ and rainfall (vertical lines):

a) 1983 , calculations carried out from $6 / 23$,

b) 1985 , calculations carried out from $6 / 26$. 

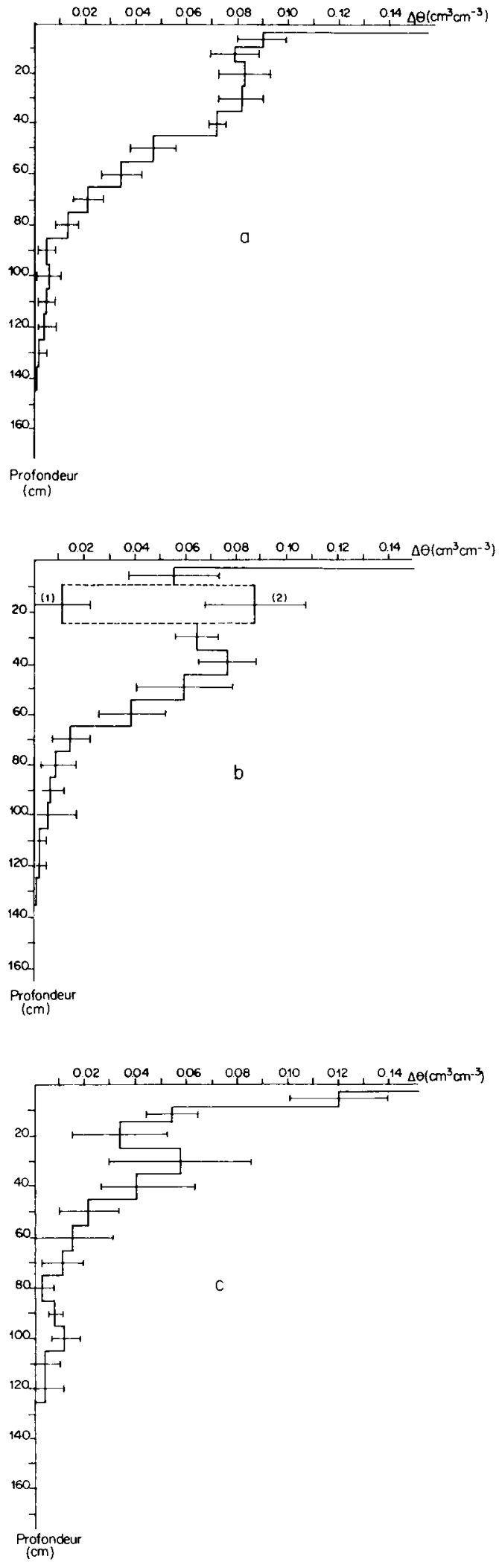

Figure 2

Variation d'humidité volumique $(\Delta \theta)$ par tranche de sol entre le 8 et le 17.7.83:

a) traitement $O$,

b) traitement $B$; état structural de la zone considérée : 1 - massif 2 - fragmentaire,

c) traitement $C$.

Variation of water volume $(\Delta \theta)$ in each soil layer between $7 / 8 / 83$ and $7 / 17 / 83$ :

a) treatment $O$,

b) treatment $B$; soil structure of the samples : 1 - massive 2 - fragmentary,

c) treatment $C$. manière nettement contrastée entre les traitements $O$ et B d'une part, et C d'autre part.

3. Le tableau 2 présente pour chaque traitement le rapport entre le terme $[\Delta \mathrm{S}]^{178}$ calculé pour les périodes sans pluie et l'ETP ${ }_{p}$ cumulée pendant ces mêmes périodes. Ce rapport est proche de 1 en $O$ et $B$; dans le traitement $C$, la variation de stock d'eau du sol n'a au contraire représenté que $2 / 3$ à $3 / 4$ de l'ETP $\mathrm{p}_{\mathrm{p}}$.

4. Les mesures tensiométriques effectuées en 1985 (fig. 3) montrent qu'aucune différence de charge hydraulique n'a été mesurée entre les profondeurs $165 \mathrm{~cm}$ et $185 \mathrm{~cm}$ pendant la période étudiée (une différence apparaît cependant par la suite, à partir du 16.8). Les remontées d'eau à travers la cote $175 \mathrm{~cm}$ ont donc probablement été de faible importance, si elles ont existé.

\section{B. Etude de la conductance stomatique}

\section{Mesures des 10, 12 et 21 juillet 1983}

Ces journées étaient claires (absence de nuages), et d'ETP $_{\mathrm{p}}$ élevée $(3,6,8,4$ et $6,2 \mathrm{~mm}$ respectivement). Les 2 premières se situent pendant la période sans pluie : le 12.7 , l'horizon $3-9 \mathrm{~cm}$ avait une humidité volumique moyenne de $0,18 \mathrm{~cm}^{3} . \mathrm{cm}^{-3}$ (fig. $5 a$ ), ce qui correspond à un potentiel de -8 bars. A la suite de la différence de vitesses d'extraction de l'eau entre les traitements, l'humidité de l'horizon $\mathrm{H} 5$ et des couches non travaillées était plus élevée en $\mathrm{C}$ qu'en $\mathrm{O}$ et $\mathrm{B}$. La $3^{\mathrm{e}}$ journée se situe après une pluie de $33 \mathrm{~mm}$ qui a ramené les horizons de surface à la capacité au champ (fig. $6 a$ ).

Lors des 2 premières journées (fig. 4 et $5 b$ ), les conductances stomatiques sont passées, en $\mathrm{O}$, par un maximum, entre 10 et $12 \mathrm{~h}$ TSV (avec un point singulier à $11 \mathrm{~h} \mathrm{le} \mathrm{12.7);} \mathrm{la} \mathrm{différence} \mathrm{entre} \mathrm{les} \mathrm{traitements} \mathrm{O}$ et B, observée à partir de $10 \mathrm{~h}$, est à la limite de la signification. Les courbes représentatives du traitement $\mathrm{C}$ se sont séparées significativement des précédentes à partir de $8 \mathrm{~h}$. A partir de $10 \mathrm{~h}$, des symptômes de flétrissement (modification de couleur et enroulement des feuilles) sont apparus sur ce traitement. Leur variabilité spatiale étant importante, nous avons distingué en C 2 types de placettes en fonction de l'intensité visuelle $\mathrm{du}$ flétrissement. Les conductances se sont différenciées sur ces 2 types de placettes, mais la forme des courbes et les niveaux atteints séparent nettement l'ensemble du traitement $\mathrm{C}$ des 2 autres.

- Le 21.7, les courbes correspondant aux 3 traitements apparaissent très proches les unes des autres (différences non significatives), et aucun symptôme de flétrissement n'est apparu (fig. 6).

2. Les résultats obtenus au cours des autres journées en 1982 et 1983 sont présentés au tableau 3. Le 8.7.83, le sol était à une humidité proche de la capacité au champ comme pour le 21.7.83 on n'a pas observé de différence entre traitements. Au contraire lorsque les couches superficielles du sol ont été partiellement desséchées $(8.7 .82$ et $12.7 .82,5.7,10.7$ et 12.7.83) une différence significative apparaît entre les traitements. En 1985, nous n'avons pas pu effectuer de mesures de conductance, mais de forts symptômes de flétrissement 


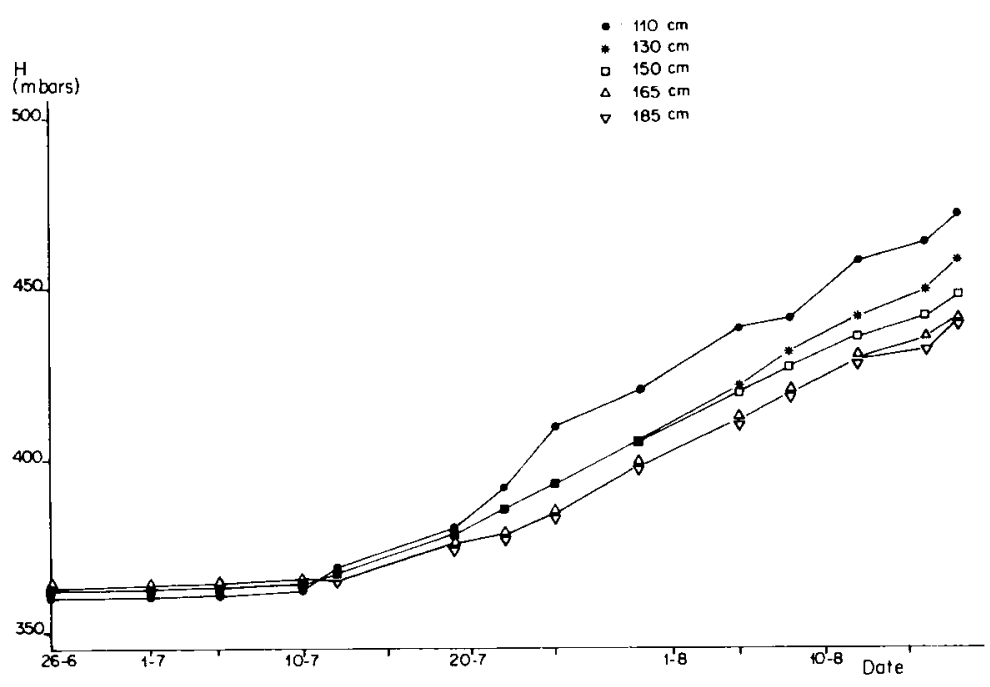

Figure 3

Evolution de la charge hydraulique entre le 26.6 et le 19.8.85.

Variation in hydraulic head from 26/6/85 to 19/8/85.

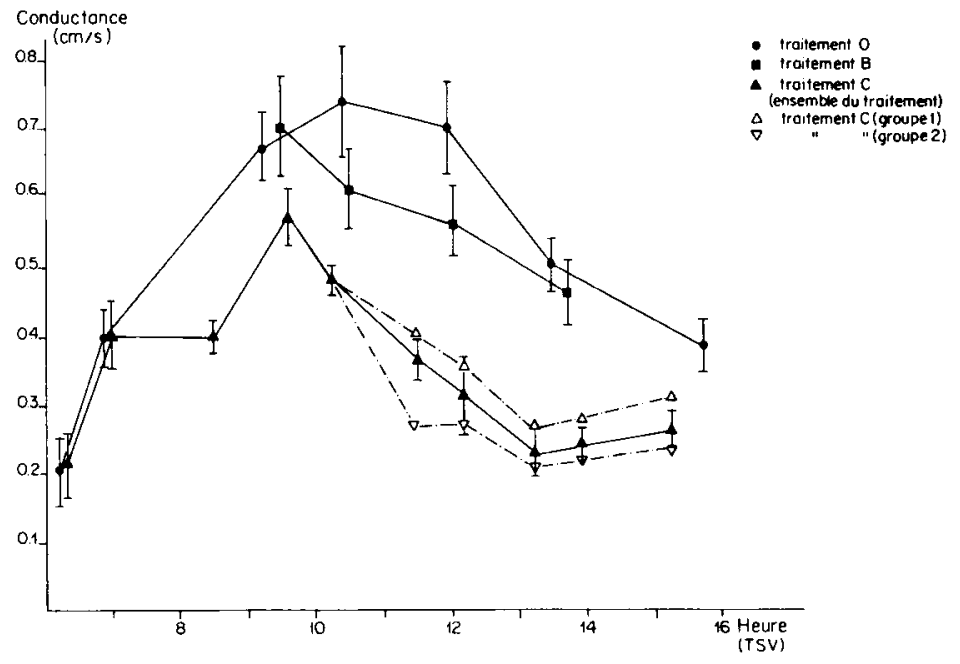

Figure 4

Conductance stomatique de la strate supérieure des couverts de mais le 10.7.83. Heures exprimées en temps solaire vrai (TSV).

Stomatal conductance of the upper maize canopy on $7 / 10 / 83$. Hours in true solar time (TSV).

ont été observés à partir du 4.7 et jusqu'au 27.7 , dans le traitement $C$ seulement. D'autre part, une différence de comportement est apparue entre les traitements $\mathrm{O}$ et $\mathrm{B}$ pendant les stades précoces (5 à 8 feuilles) : des symptômes de flétrissement ont été observés en 1982 et 1983 dans le traitement B pendant cette période, et les conductances mesurées le 20.6 .83 (stade 8 feuilles) sont significativement différentes entre $\mathrm{O}$ et $\mathrm{B}$.

\section{Mise en correspondance avec les mesures d'enraci- nement et de surface foliaire.}

\section{Variations de stock d'eau du sol}

Le classement entre traitements du terme $[\Delta S]_{0}^{175}$ est conforme à celui établi (TARDIEU \& MANICHON, 1987b) à partir de l'indice synthétique de caractérisa- tion des enracinements. Mais il suit aussi le classement des indices foliaires, qui sont de $3.4,2.5$ et 2.0 respectivement en $\mathrm{O}, \mathrm{B}$ et $\mathrm{C}$ en 1983, et de 3.2 et 2.0 en $\mathrm{O}$ et $\mathrm{C}$ en 1985. La variation de stock d'eau du sol est donc la plus grande dans le traitement $O$, où les capteurs et les évaporateurs d'eau sont les plus développés.

\section{Conductance stomatique}

A l'intérieur de chaque groupe de placettes, celle-ci est fortement liée à l'indice synthétique de caractérisation de l'enracinement (fig. 7). Cette liaison positive n'apparaît qu'à partir de $8 \mathrm{~h} 15$ TSV, et est maximale à $11 \mathrm{~h} 45$. Elle rend compte de la variabilité spatiale de la conductance stomatique et des symptômes de flétrissement dans le traitement $\mathrm{C}$, qui avait abouti à distinguer 2 types de placettes. D'autre part, la différence entre 

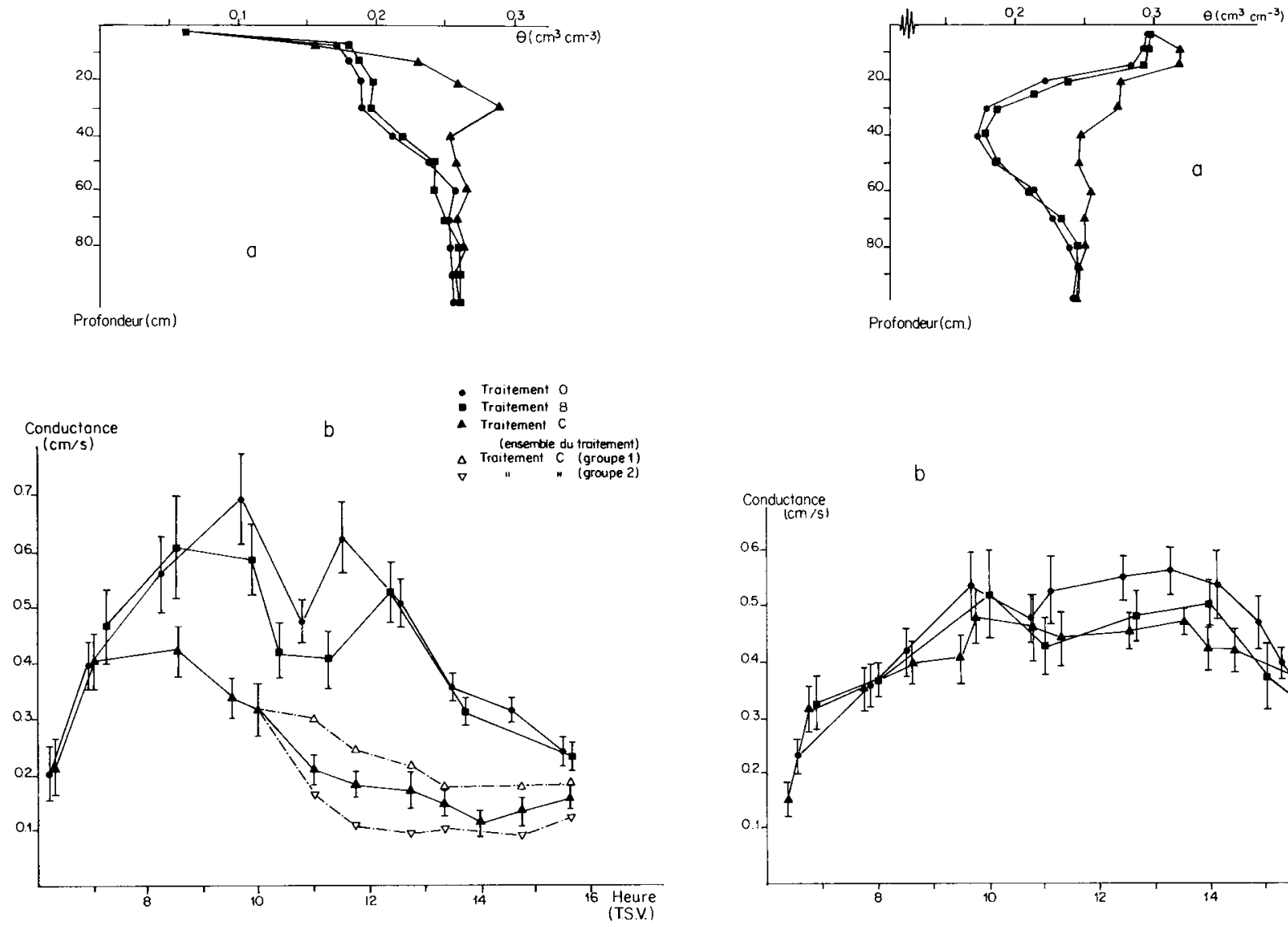

Figure 5

Humidité volumique du sol (a) et conductance stomatique (b) mesurées le 12.7.83.

Measurements of soil water volume (a) and stomatal conductance (b) on $7 / 12 / 83$.

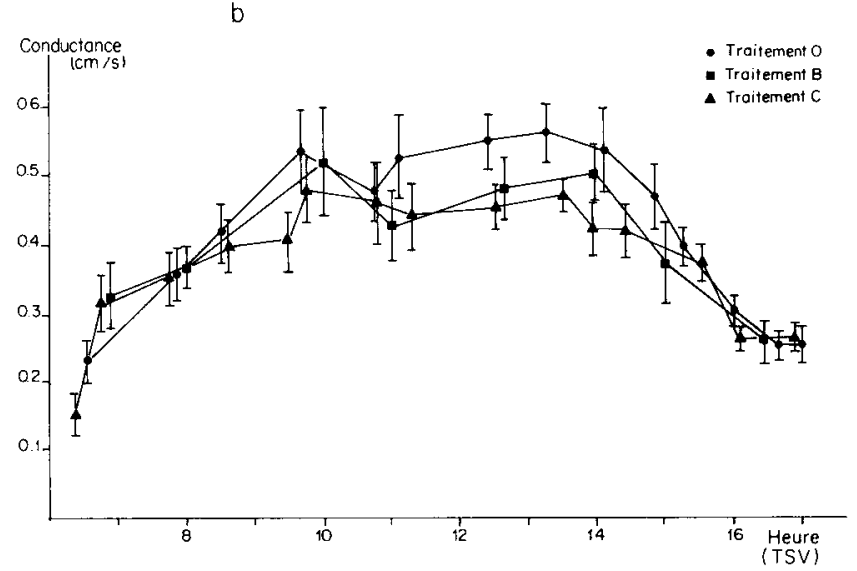

Figure 6

Humidité volumique du sol (a) et conductance stomatique (b) mesurées le 21.7.83.

Measurements of soil water volume (a) and stomatal conductance (b) on the $7 / 12 / 83$.

TABLEAU 3

Mesures de conductance stomatique et observation de symptômes de flétrissement, 1982 et 1983. «> » représente une différence significative à $0,05$. Stomatal conductance measurements and observations on wilting symptoms, 1982 and 1983. "> " means a significant difference at 0.05 .

\begin{tabular}{|c|c|c|c|c|c|}
\hline \multirow{2}{*}{ Date } & \multirow{2}{*}{$\begin{array}{c}\operatorname{ETP}_{p} \\
\text { de la } \\
\text { journée }\end{array}$} & \multirow{2}{*}{$\begin{array}{c}\mathrm{ETP}_{\mathrm{p}} \text { cumulée } \\
\text { depuis le début } \\
\text { de la période } \\
\text { sèche }\end{array}$} & \multirow{2}{*}{$\begin{array}{l}\text { Différences } \\
\text { observées }\end{array}$} & \multicolumn{2}{|c|}{$\begin{array}{l}\text { Heures d'apparition des différences } \\
\text { entre traitements }\end{array}$} \\
\hline & & & & $\begin{array}{c}\text { de } \\
\text { conductance }\end{array}$ & $\begin{array}{l}\text { de symptomes } \\
\text { de flétrissement }\end{array}$ \\
\hline \multicolumn{6}{|l|}{1983} \\
\hline 20.06 & 4.9 & 38.5 & $\mathrm{O}>\mathrm{B}>\mathrm{C}$ & $8 \mathrm{~h}$ & $12 \mathrm{~h}$ \\
\hline 5.07 & 5.2 & 13.5 & $\mathrm{O}=\mathrm{B}>\mathrm{C}$ & $9 \mathrm{~h}$ & $10 \mathrm{~h}$ \\
\hline 8.07 & 3.5 & 6,3 & $\mathrm{O}=\mathrm{B}=\mathrm{C}$ & - & - \\
\hline 10.07 & 3.6 & 13.9 & $\mathrm{O}=\mathrm{B}>\mathrm{C}$ & $8 \mathrm{~h}$ & 10 h 30 \\
\hline 12.07 & 8.4 & 31.1 & $\mathrm{O}=\mathrm{B}>\mathrm{C}$ & $8 \mathrm{~h}$ & 10 h 30 \\
\hline 21.07 & 6.2 & 5.6 & $\mathrm{O}=\mathrm{B}=\mathrm{C}$ & - & - \\
\hline \multicolumn{6}{|l|}{1982} \\
\hline 8.07 & 5.0 & 15.1 & $\mathrm{O}=\mathrm{B}>\mathrm{C}$ & $9 \mathrm{~h}$ & $10 \mathrm{~h}$ \\
\hline 12.07 & 5.0 & 31.3 & $\mathrm{O}>\mathrm{B}>\mathrm{C}$ & $9 \mathrm{~h}$ & $11 \mathrm{~h}$ \\
\hline
\end{tabular}

les traitements $\mathrm{O}$ et $\mathrm{B}$ en début de végétation est en accord avec les faibles valeurs de l'indice synthétique en B à cette période (TARDIEU \& MANICHON, 1987b).

\section{Variabilité spatiale du dessèchement du sol}

Cette variabilité peut être mise en correspondance avec les caractéristiques géométriques des systèmes racinaires :
- L'horizon 0-15 cm, dont tous les points sont situés à proximité d'une racine quel que soit le traitement, est desséché d'une manière peu différente dans chacun de ceux-ci. La tendance vers un dessèchement plus faible en B pourrait être due au fort degré de groupement des racines et aux défauts de contact entre celles-ci et la terre environnante.

- Dans l'horizon H5 du traitement B, une part importante de la variabilité spatiale de l'humidité à la 


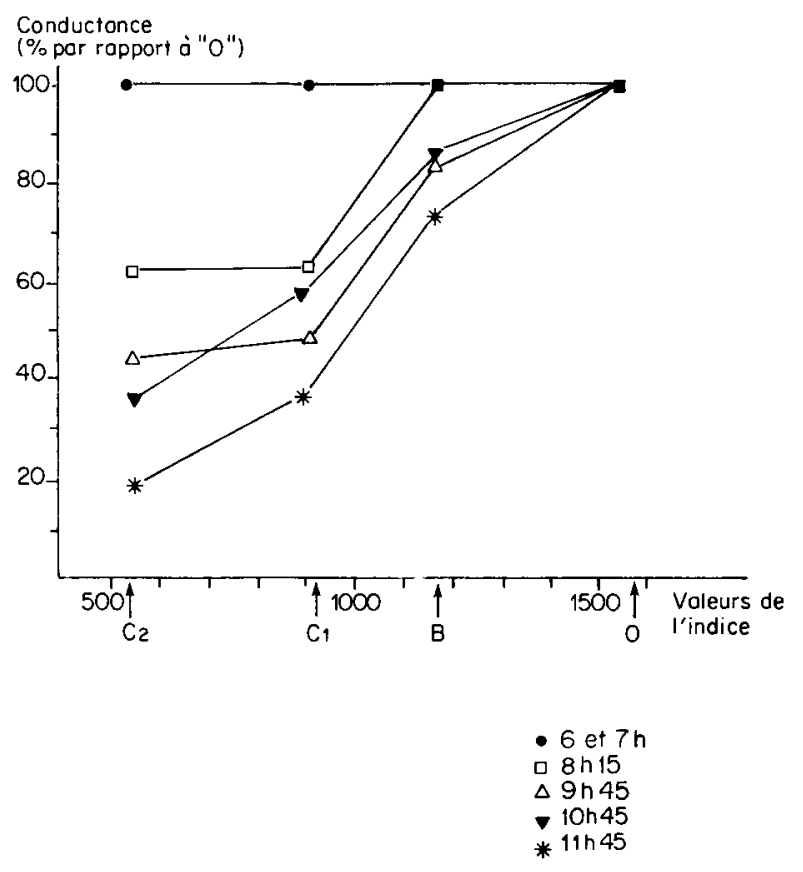

Figure 7

Relation entre les indices synthétiques de caractérisation de l'euracinement et les conductances stomatiques mesurées le 12.7.83 (exprimées à chaque heure de la journée en pourcentage de la valeur mesurée en $O$ ).

Synthetic index of characterization of the root system plotted against stomatal conductances measured on 7/12/83 (in percent of the value measured at $O$ ).

fin des périodes sans pluies est liée à la différence de vitesse de dessèchement entre zones massives non enracinées et zones fragmentaires enracinées. Le 13.7.1983, les premières avaient une humidité volumique moyenne de $0,29 \mathrm{~cm}^{3} \cdot \mathrm{cm}^{-3}$ (correspondant à un potentiel hydrique de 1,2 bars), alors que celle des secondes était de $18,7 \mathrm{~cm}^{3} . \mathrm{cm}^{-3}$ ( 7 bars). Cette différence de potentiel a été mesurée sur des distances de l'ordre du dm; des différences similaires avaient été observées lors d'une $1^{\text {re }}$ période sans pluie de la même année (TARDIEU \& MANICHON, 1987a).

- Dans l'horizon H5 et les couches sous-jacentes, où la densité racinaire est nettement supéricure en $O$ et $B$ qu'en $C$, la variation de stock d'eau est classée de la même manière entre traitements. Cependant, la différence de densité ne peut pas expliquer entièrement cette différence de comportement : dans les traitements $\mathrm{O}$ et $\mathrm{C}$ nous disposons conjointement du nombre d'impacts de racines sur 5 plans horizontaux (numériquement égal à la longueur de racines par unité de volume du voisinage des plans étudiés), et de la variation de stock d'eau au voisinage de ces plans, ces 2 variables ont été mises en correspondance (tabl. 4). Leur rapport, qui peut être assimilé au flux d'eau par unité de longueur de racine, apparaît plus élevé en $O$ qu'en $C$, alors qu'on pouvait attendre un résultat inverse (cf. discussion).

4. Comparaison des profondeurs de dessèchement du sol et d'enracinement

$\mathrm{Au}$ cours des 2 périodes sans pluie, la profondeur à laquelle des variations d'humidité ont été constatées (fig. 2) est nettement supérieure à la profondeur d'enracinement, inférieure à $1,10 \mathrm{~m}$ dans tous les traitements. Entre le 26.6 et le 3.10 .85 , la variation de stock d'eau dans la couche $115-175 \mathrm{~cm}$ est de $18,3 \mathrm{~mm}$. Ces observations sont convergentes avec celles de DAUDET \& VALANGOGNE (1976) et de KATERJI et al. (1984) sur la contribution des couches profondes du sol au bilan hydrique.

\section{DISCUSSION}

\section{A. Existence d'un déficit hydrique lié à l'état structu- ral de la couche labourée}

\section{Intégration au niveau de la journée}

Pendant des journées claires, la forme et les valeurs atteintes par la courbe de variation horaire de la conductance stomatique sont des indicateurs de l'intensité des stress hydriques (BERGER, 1973 ; TURNER, 1974) : en alimentation hydrique optimale, la variation dans le temps de la conductance suit celle de l'éclairement, le maximum se situant à midi solaire ; dans le cas contraire la conductance cesse d'augmenter à une heure d'autant plus précoce que le stress est intense.

Le premier type de courbe est observé dans les 3 traitements lorsque les $1^{\text {er }} \mathrm{cm}$ du sol sont humides. Les systèmes racinaires indépendamment de leurs caractéristiques géométriques, ont alors fourni un flux d'eau suffisant par rapport à la demande climatique. Ce flux provient essentiellement de l'horizon $0-15 \mathrm{~cm}$, et (vraisemblablement) des zones les plus proches des racines dans les horizons plus profonds. Ce type de courbe est également observé, quelle que soit la date, dans le traitement $\mathrm{O}$. La baisse de conductance survenue en $\mathrm{O}$ le 12.7 à $13 \mathrm{~h}$ peut être interprétée comme une courte régulation stomatique (il n'y a pas eu de passage nuageux à ce moment). On ne peut cependant pas totalement rejeter la possibilité d'une modification passagère de l'étalonnage de l'appareil.

Lorsque le potentiel hydrique de l'horizon $\mathrm{Hl}$ s'est abaissé, le flux d'eau nécessaire au maintien de l'ouverture stomatique provient surtout de l'horizon $\mathrm{H} 5$ et des horizons non travaillés, différemment colonisés entre traitements. On observe alors un décrochement de la conductance stomatique dans le traitement $\mathrm{C}$ et pour certaines dates dans le traitement $\mathrm{B}$, par rapport à $\mathrm{O}$; l'heure d'apparition et l'intensité du stress hydrique sont dans ce cas en étroite relation avec les caractéristiques géométriques de l'enracinement caractérisées par l'indice synthétique. Il est à noter que ce stress n'est apparu que dans les parcelles d'état structural le plus défavorable, bien que les conditions climatiques soient les mêmes sur l'ensemble du dispositif, et que ces parcelles soient celles où le sol est le plus humide puisque le stock d'eau y a été moins capté par le système racinaire (fig. 5a). Ceci fait apparaître une sérieuse limitation aux méthodes de déclenchement d'irrigation fondées sur la mesure de l'humidité ou du potentiel du sol. Pour des pas de temps allant de la mn à la journée, les différences entre caractéristiques géométriques des systèmes racinaires ont donc eu des conséquences importantes sur l'alimentation hydrique des peuplements de maïs étudié. 
TABLEAU 4

Densités d'impacts de racines $(N)$ sur les plans horizontaux situés à $17,30,60$ et $80 \mathrm{~cm}$ de profondeur, et écarts d'humidité volumique ( $\Delta \theta$ ) mesurés aux mêmes profondeurs entre les 8 et 17.7.83. (Les nombres entre parenthèses représentent les demi-distances moyennes entre racines voisines calculées à partir des densités d'impacts.)

Root impact densities on horizontal 17,30,60 and $80 \mathrm{~cm}$ deep planes, and water volume differences $(\Delta \theta)$ measured at the same depths between 8 and $7 / 17 / 83$. (Numbers in brackets represent half-distances between neighbouring roots calculated from root impact densities.)

\begin{tabular}{ccccccc}
\hline \hline & \multicolumn{2}{c}{ Traitement $\mathrm{O}$} & \multicolumn{2}{c}{ Traitement C } \\
\cline { 2 - 6 } Profondeur & $\begin{array}{c}\text { Nombre } \\
\text { d'impacts } \\
\text { par dm }\end{array}$ & $\begin{array}{c}\Delta \theta \\
\mathrm{cm}^{3} \cdot \mathrm{cm}^{-3} \\
\times 100\end{array}$ & $\begin{array}{c}\Delta \theta \\
\mathrm{N}\end{array}$ & $\begin{array}{c}\text { Nombre } \\
\text { d'impacts } \\
\text { par dm }\end{array}$ & $\begin{array}{c}\Delta \theta \\
\mathrm{cm}^{3} \cdot \mathrm{cm}^{-3} \\
\times 100\end{array}$ & $\begin{array}{c}\Delta \theta \\
\mathrm{N}\end{array}$ \\
\hline $17 \mathrm{~cm}$ & $49,0(0,76)$ & 8,3 & 0,17 & $38,7(0,86)$ & 3,4 & 0,09 \\
$30 \mathrm{~cm}$ & $23,3(1,11)$ & 7,2 & 0,31 & $15,9(1,34)$ & 4,0 & 0,25 \\
$60 \mathrm{~cm}$ & $14,0(1,43)$ & 3,4 & 0,24 & $7,9(1,90)$ & 1,5 & 0,19 \\
$80 \mathrm{~cm}$ & $6,1(2,17)$ & 1,3 & 0,21 & $3,9(2,71)$ & 0,3 & 0,08 \\
\hline \hline
\end{tabular}

\section{Intégration sur la décade}

Cette conclusion peut être étendue à une période plus longue par la comparaison entre l'ETP $_{p}$ et la variation du stock d'eau du sol. Pendant les périodes sans pluie et en l'absence de remontées importantes d'eau à travers la cote $175 \mathrm{~cm}$, cette dernière peut être assimilée à l'ETR. Elle est très proche de l'ETP ${ }_{p}$ dans les parcelles d'état structural favorable (résultat semblable à celui de VACHAUD et al., 1985), mais lui est nettement inférieure dans le traitement $C$ pendant les 2 périodes sans pluies étudiées. Les indices foliaires étant supérieurs à 2 dans tous les traitements, il est peu probable que cette différence d'évapotranspiration entre couverts végétaux soit due aux écarts de surface foliaire (PERRIER, 1977) ; on peut donc l'attribuer à la qualité des capteurs d'eau.

\section{B. Transferts d'eau sol-plante et disposition spatiale des racines}

Bien que la profondeur d'enracinement soit proche entre traitements et que la "réserve utile " soit la même sur l'ensemble du champ expérimental, des écarts appréciables de vitesse d'utilisation du stock d'eau par les racines ont été constatés. Ceci ne pouvait pas être prévu à partir des modèles de transferts d'eau évoqués en introduction : les demi-distances moyennes entre racines voisines calculées à partir des nombres d'impact par unité de surface (TARDIEU \& MANICHON, 1986) sont inférieures à $2 \mathrm{~cm}$ en $O$ et $C$, jusqu'à $60 \mathrm{~cm}$ de profondeur ; d'après le modèle de GARDNER (1960) et les calculs de NEWMAN (1969), elles ne peuvent provoquer de résistance importante au transfert d'eau ni dans le traitement $O$, ni dans le traitement $C$. L'existence d'un stress hydrique en $\mathrm{C}$ à une période où le potentiel du sol ne s'est pas encore fortement abaissé ne peut s'expliquer que si on prend en compte la disposition spatiale des racines. En $\mathrm{C}$, près de la moitié des points de $\mathrm{H} 5$ et un quart de l'horizon $0-60 \mathrm{~cm}$ se trouve à une distance supérieure à $4 \mathrm{~cm}$ de la racine la plus proche, ce qui n'est pas le cas en O (TARDIEU \& MANICHON, 1987b). Il est donc très vraisemblable que cette disposition, plus irrégulière en $\mathrm{B}$ et $\mathrm{C}$ qu'en $\mathrm{O}$, a eu un effet important sur les résistances au transfert de l'eau entre le sol et les racines. Deux faits tendent à renforcer cette conclusion :

- Il existe des différences de potentiel hydrique de l'ordre de 6 bars sur quelques $\mathrm{cm}$, entre zones enraci- nées et non enracinées voisines de l'horizon $\mathrm{H} 5$ de $\mathrm{B}$. Ces gradients sont du même ordre de grandeur que ceux mesurés par DUNHAM \& NYE (1973) en conditions contrôlées, à des distances croissantes d'un plan enraciné.

- L'absorption d'eau par unité de longueur de racine est plus grande en $\mathrm{O}$ qu'en $\mathrm{C}$ (tabl. 4) bien que la densité racinaire soit plus faible en $\mathrm{C}$ et que l'on pourrait, de ce fait, $\mathrm{y}$ attendre une plus faible compétition entre racines pour l'absorption d'eau. Ce résultat peut être comparé à ceux de BALDWIN et al. (1972), qui à l'aide d'une simulation basée sur un analogue électrique, suggèrent que pour une même densité racinaire, l'efficacité d'un enracinement est d'autant plus réduite que la disposition des racines est plus groupée. Il est également cohérent avec les calculs de PASSIOURA (1985) relatifs à l'effet de la disposition des racines sur leur absorption.

\section{CONCLUSION}

L'état structural de la couche labourée a une forte influence sur la capacité des systèmes racinaires à extraire l'eau du sol. Cela se traduit dans les parcelles d'état structural le plus défavorable par une moindre utilisation des réserves en eau et par une plus grande fréquence de stress hydriques, qui peuvent s'y produire même quand la majeure partie de la zone enracinée est encore humide. Les peuplements de maïs du traitement $\mathrm{C}$ apparaissent comme les plus sensibles aux variations climatiques pendant la "période critique " : des sécheresses de plusieurs jours y ont eu pour conséquences des stress hydriques répétés, ce qui n'a pas été le cas dans les parcelles d'état structural favorable. D'autre part, les critères de caractérisation de l'enracinement présentés dans l'article précédent de cette série ont permis d'expliquer une part importante de la variabilité spatiale du dessèchement du sol, entre parcelles expérimentales, mais aussi à l'intérieur de celles-ci entre zones voisines de la couche labourée. Ceci renforce l'idée, émise dans un article précédent (TARDIEU \& MANICHON, 1986) que la disposition spatiale des racines est un critère pertinent pour caractériser l'enracinement en tant que capteur d'eau. 


\section{RÉFÉRENCES BIBLIOGRAPHIQUES}

Baldwin J. P., Tinker P. B., Nye P. H., 1972. Uptake of solutes by multiple root systems from soil. II. - The theoretical effects of rooting density and pattern on uptake of nutrients from soil. Plant and soil, 36, 693-708.

Berger A., 1973. Le potentiel hydrique et la résistance à la diffusion dans les stomates, indicateurs de l'état hydrique de la plante. In Plant response to climatic factor. Proceedings of Uppsala Symposium., 1979. SLAYTER ed., pp. 201-212. UNESCO, Paris, 574 p.

Bouchet R. J., Robelin M., 1969. Evaporation potentielle et réelle. Domaine d'utilisation portée pratique. Bull. tech. Inf., 238, 215-230.

Claasen M. M., Shaw R. H., 1979. Water deficit effects on corn. I. - Vegetative components ; II. - Grain components. Agron. J., 62, 649-655.

Daudet F. A., Valancogne Ch., 1976. Mesure des flux profonds de drainage ou de remontées capillaires. Leur importance dans le bilan hydrique. Ann. agron., 27, 165-182.

Daudet F. A., Vachaud G., 1977. La mesure neutronique du stock d'eau du sol et ses variations. Application à la détermination du bilan hydrique. Ann. agron., 28, 503-519.

Denmead O. T., Shaw R. H., 1960. The effect of plant moisture stress at different stages of growth on the development and yield of corn. Agron. J., 52, 272-274.

Dunham R. J., Nye P. H., 1973. The influence of water content on the uptake of ions by roots. I. - Soil water content gradient near a plane of onion roots. J. appl. Ecol., 10, 585-598.

Gardner W. R., 1960. Dynamic aspect of water availability to plants. Soil Sci., 89, 63-73.

Katerji N., Oulid-Aissa A. K., 1983. Etude et amélioration de la fiabilité d'un poromètre à diffusion utilisé en milieu naturel. Agronomie, 3, 931-936.

Katerji N., Perrier A., Oulid-Aissa A. K., 1983. Exploration au champ et interprétation de la variation horizontale et verticale de la résistance stomatique : cas d'une culture de luzerne. Agronomie, 3, 847-855.

Katerji N., Daudet F., Valancogne Ch., 1984. Contribution des réserves profondes du sol au bilan hydrique des cultures. Détermination et importance. Agronomie, 4, 779-787.

Manichon H., Sebillotte M., 1973. Etude de la monoculture de maïs. Résultats d'enquêtes agronomiques en Béarn. Doc. ronéo. Chaire d'Agronomie, INA-PG, Paris, 108 p.

Newman E. I., 1969. Resistance to water flow in soil and plant. I. Soil resistance in relation to amounts of root : theoretical estimates. II. - A review of experimental evidence on the rhizosphere resistance, J. appl. Eco., 6, 1-12 et 6, 261-272.

Passioura J. B., 1985. Roots and water economy of wheat. In "Wheat growth and modelling", W. DAY and R. K. ATKIN Ed., NATO ASI Series : 1985, Plenum Press, New York, 407 p.
Perrier A., 1977. Etude des variations de l'évapotranspiration à partir d'un modèle analytique prenant en compte les variations verticales des densités de surface foliaire et des résistances stomatiques. $L a$ Météorologie, 6, 41-46.

Raghavan G. S. V., Mc Kyes E., Baxter R., Gendron G., 1979. Traffic-soil-plant (maize) relations. J. Terramechanics, 16, 181-189.

Reicosky D. C., Ritchie J. T., 1976. Relative importance of soil resistance and plant resistance in root water absorption. Soil Sci. Soc. Am. J., 40, 293-297.

Robelin M., 1963. Contribution à l'étude du comportement du maïs grain vis-à-vis de la sécheresse. In "Journées Intern. Irrig. AGPM. Pau'. 69-76, AGPM, Paris.

Sanchez-Diaz M., Kramer P. J., 1971. Behavior of corn and sorghum under water stress and during recovery. Plant physiol., 48, 613616.

Tardieu F., 1984. Etude au champ de l'enracinement du maïs. Influence de l'état structural sur la répartition des racines. Conséquences pour l'alimentation hydrique. Thèse de Docteur Ingénieur. INA-PG, Paris, 232 p.

Tardieu F., Manichon H., 1986. Caractérisation en tant que capteur d'eau de l'enracinement du maïs en parcelle cultivée. I. - Discussion des critères d'étude. Agronomie, 6 (4), 345-354.

Tardieu F., Manichon H., 1987a. Etat structural, enracinement et alimentation hydrique du maïs. I. - Modélisation d'états structuraux types de la couche labourée. Agronomie, 7 (2), 51-59.

Tardieu F., Manichon H., 1987b. Etat structural, enracinement et alimentation hydrique du maïs. II. - Croissance et disposition spatiale du système racinaire. Agronomie, 7 (3), 201-211.

Turner N. C., 1974. Stomatal behavior and water status of maize, sorghum and tobacco under field conditions. II. - At low soil potential. Plant Physiol., 53, 360-365.

Vachaud G., Vauclin M., Riou Ch., Chaabouni Z., 1985. Evaporation en zone semi-aride de 2 couverts végétaux (gazon, blé) obtenue par plusieurs méthodes. II. - Méthodes neutroniques et tensiométriques. Agronomie, 5, 267-274.

Van Loon C. D., Bouma J., 1978. A case study on the effect of soil compaction on potato growth in a loamy sand soil. 2 Potato plant responses. Neth. J. agri. Sci., 26, 421-429.

Vauclin M., Haverkamp R., Vachaud G., 1984. Error analysis in estimating soil water content from neutron probe measurements $: 2$. Spatial stand point. Soil Sci., 137, 141-148.

Zur B., Jones J. W., Boote K. J., Hammond L. C., 1982. Total resistance to water flow in field soybeans. II. - Limiting soil moisture. Agron. J., 74, 99-105. 